\title{
Hacia la universidad digital: ¿dónde estamos y a dónde vamos?
}

\section{Towards the digital university: where are we and where are we going?}

\author{
Manuel Area Moreira \\ Universidad de La Laguna \\ Coordinador del Monográfico
}

\section{Resumen}

Las instituciones universitarias se encuentran actualmente en una etapa de tránsito de un modelo de enseñanza decimonónico basado con la transmisión del conocimiento empaquetado en objetos culturales impresos y en la lección magistral del docente hacia un modelo pedagógico apoyado en el aprendizaje activo y constructivo del alumnado interaccionando con recursos digitales. Este número monográfico aborda el análisis de la transformación digital de la universidad guiado por cuestiones como ¿Hacia dónde debiera evolucionar la educación universitaria? ¿Qué sentido pedagógico tiene la entrada de la digitalización en la enseñanza universitaria? ¿Cómo transforma la tecnología la profesionalidad docente, lo que debe aprender el alumnado, o los entornos y las metodologías de eLearning?

Palabras clave: universidad digital; TIC; docencia virtual; tecnología; eLearning.

\begin{abstract}
Universities are currently undergoing the transition from a conventional teaching model based on knowledge transfer through lecturing and printed cultural objects, towards a pedagogical model based on student active and constructive learning and their interaction with digital resources. This special issue addresses the analysis of universities' digital transformation guided by questions such as: Where should university education evolve to? Which is the pedagogical interpretation of digitalization in university education? How does technology transform teaching professionalism, what students should learn, or the environments and methodologies of eLearning?
\end{abstract}

Keywords: digital university; ICT; virtual teaching; technology; eLearning.

La universidad es una institución educativa antigua. Han pasado más de ocho siglos desde su fundación a finales de la Edad Media ${ }^{1}$ existiendo actualmente más de 13.000 universidades catalogadas en el mundo² lo que evidencia que, además 
de su longevidad, los centros universitarios siguen siendo instituciones educativas altamente demandadas en cualquier parte del planeta. Seguramente la razón de esta supervivencia viene dada porque la universidad es un foco de creación, formación y difusión de conocimiento imprescindible para el avance social, cultural y económico de las sociedades modernas. Desde la Ilustración, y sobre todo con la sociedad industrial, las universidades han sido centros de estudios superiores para la formación de los dirigentes, de los técnicos, de los creadores, de los empresarios, de los profesionales y de la ciudadanía en general de un país o Estado.

En la sociedad del conocimiento, la necesidad de educación superior todavía es más necesaria porque el saber y competencia humana será valiosa en una sociedad automatizada digitalmente. Avanzamos hacia la adaptación o transformación de la educación superior en una civilización o estadio de la humanidad que vive en un ecosistema digital. ¿Hacia dónde debiera evolucionar la educación universitaria? ¿Qué sentido pedagógico tiene la entrada de la digitalización en la enseñanza universitaria? ¿Cómo transforma la tecnología la profesionalidad docente, lo que debe aprender el alumnado, o los entornos y las metodologías de eLearning? Éstas son algunas de las preguntas que nos formulamos en este número especial de celebración de los 30 años de RIED.

Es necesario recordar que durante muchos siglos los estudios universitarios fueron un escenario formativo restringido para minorías que disponían de la renta económica, del privilegio social o del nivel cultural que les permitían acceder a las titulaciones de educación superior. Pero, afortunadamente, los tiempos han cambiado.

Desde mediados del siglo pasado se inició un proceso de apertura y democratización en la entrada del alumnado a los estudios universitarios dejando de ser algo exclusivo para un sector minoritario de la población. La expansión de la clase media, el desarrollo de la sociedad industrial y del bienestar, el crecimiento del sector servicios, el aumento y concentración de la población en urbes en detrimento de los núcleos rurales, las políticas de redistribución de la riqueza y de compensación de desequilibrios económicos, ..., junto con la demanda de profesionales de alto nivel de estudios favoreció que la educación superior en el último tercio del siglo XX empezara a ser una formación accesible a sujetos de diferentes clases sociales.

Estamos en un tiempo acelerado. Hace pocos años decíamos que nuestro presente era una época caracterizada por la tercera revolución industrial provocada de las telecomunicaciones y de la informática. Ahora se habla de la cuarta revolución basada en la automatización del conocimiento, del trabajo y de los procesos sociales. Es decir, el futuro parece ser un tiempo dominado por las máquinas digitales y su lógica de funcionamiento (Castell, 1999; Postman, 1994).

$\mathrm{El}$ incremento exponencial en la producción e intercambio de información; el acelerado desarrollo de las comunicaciones interpersonales a través de las redes sociales; la tecnificación y automatización de los procesos de gestión informacional y organizacional de las instituciones y empresas; los cambios en las reglas y mecanismos 
de generación, difusión, acceso y consumo de conocimiento; la aparición de nuevas interfaces, formatos y lenguajes en la codificación y representación de la información (hipertextos, transmedia, multimedia, realidad aumentada, realidad virtual, memes, ...); las transformaciones culturales de las generaciones más jóvenes como son los Millennials, entre otros muchos fenómenos más, está provocando que los modelos y formas tradicionales de enseñanza universitaria basada en la clase magistral, en los apuntes, en el manual de estudio y en los exámenes hayan entrado en crisis, siendo cuestionados, no sólo por los estudiantes, sino también por las instituciones, los expertos y los docentes.

Todo este conjunto de fenómenos configuran un contexto o coyuntura sociocultural que reclama no solo la incorporación de las tecnologías digitales a la enseñanza universitaria (tanto en la modalidad presencial como a distancia), sino también profundos cambios metodológicos y organizativos en el modo de gestionar el tiempo, los espacios, la profesionalidad docente, los contenidos que se enseñan, las actividades de aprendizaje, la evaluación y las formas de comunicarnos con el alumnado. Los retos y desafíos que implican la sociedad informacional o digital a las instituciones universitarias son profundos, complejos y de largo alcance. Sobre este particular existen numerosas obras, estudios e informes que están analizando este proceso de transformación y readaptación de la educación superior a las nuevas exigencias de la sociedad del siglo XXI que el lector encontrará sin grandes dificultades en la Red.

Existe consenso en el discurso teórico en superar el paradigma pedagógico tradicional o de enseñanza expositiva y aprendizaje por recepción. Esta concepción y práctica educativa está íntimamente arraigada en la genética docente universitaria desde sus orígenes y, aunque en el actual discurso oficial o retórica dominante se cuestione, su presencia real sigue vigente en la mayoría de las aulas tanto presenciales como las denominadas virtuales o en línea. Pero también es cierto, que de modo creciente, existen docentes innovadores, experiencias y proyectos de innovación docente universitaria empleando las TIC que se apoyan y desarrollan modelos pedagógicos basados en procesos de aprendizaje activos, que requieren al alumnado, la búsqueda, análisis, construcción y comunicación del conocimiento y no su mera reproducción. El panorama, en este sentido, es altamente interesante y halagüeño.

Muchos de los autores que firmamos los artículos de este monográfico pertenecemos, en mayor o menor medida temporal, a una generación académica que tuvo la oportunidad de ser pionera en explorar los usos incipientes de los ordenadores y de Internet aplicados a la enseñanza universitaria en la última década del siglo XX. En este sentido, pudiéramos afirmar, que somos coetáneos con el nacimiento de la revista RIED y a lo largo de estos últimos 30 años hemos sido a la vez, tanto espectadores como participantes activos en la aplicación educativa de las distintas tecnologías que han ido sucediéndose desde entonces: el multimedia, los videojuegos, el hipertexto, las actividades interactivas, las web docentes, los LMS y aulas virtuales, y más recientemente el conjunto variado de tecnologías inalámbricas 
o móviles, las aplicaciones de la web 2.0, de la realidad virtual, los cursos MOOC y las redes sociales.

Los firmantes de los artículos de este monográfico Hacia la universidad digital: ¿dónde estamos y a dónde vamos? compartimos unas mismas coordenadas o premisas teóricas que, inspirados en el análisis de la sociedad informacional, la pedagogía de la Escuela Nueva y en las aportaciones de la psicología del aprendizaje quieren impulsar un cambio de paradigma pedagógico con relación a la docencia universitaria en la sociedad digital.

En este número se abordan algunos de los tópicos y problemas que actualmente estamos investigando desde lo que pudiera considerarse la comunidad académica de la Tecnología Educativa en el ámbito iberoamericano. Por ello, en este monográfico se entremezclan tanto ensayos o trabajos de corte teórico y reflexivo sobre hacia dónde debe evolucionar la universidad digital con otros artículos de estudios empíricos sobre esta problemática. Todos ellos son piezas de un puzle complejo que persigue construir un discurso racional y académico sobre la educación superior en la sociedad digital.

Comienza este monográfico con el ensayo de J. M. Sancho A. Ornellas, y J. Arrazola de la Universidad de Barcelona titulado La situación cambiante de universidad en la era digital. En el mismo, las autoras realizan un análisis de cómo la evolución de las tecnologías está afectando a la transformación de la profesionalidad docente universitaria y concluyendo sobre los retos y perspectivas universitarias actuales, a partir de distintos estudios y propuestas internacionales.

El siguiente artículo es un ensayo firmado por Adell, Castañeda y Esteve de la Universidad Jaume I titulado ¿Hacia la Ubersidad? Conflictos y contradicciones de la universidad digital donde analizan el proceso y efectos de la incorporación de las tecnologías digitales a la docencia e investigación universitaria desde posicionamientos del pensamiento crítico.

La profesora de la Universidad de Barcelona, G. Gros, en su artículo titulado $L a$ evolución del e-learning: del aula virtual a la red desarrolla una síntesis teórica en torno al concepto de aprendizaje en línea o eLearning abordando dimensiones como su evolución tecno-pedagógica, la mezcla de los espacios presenciales y virtuales, el impacto o eficacia de esta modalidad educativa, y lo que son los desarrollos de la automatización en el elearning (espacios personales de aprendizaje o PLE, tutores o agentes digitales y las analíticas del aprendizaje).

El trabajo firmado por J. De Pablos profesor de la Universidad de Sevilla titulado Las tecnologías digitales y su impacto en la Universidad. Las nuevas mediaciones es un ensayo donde analiza, desde la teoría de la mediación, cómo la evolución de las tecnologías desde las tradicionales hacia las digitales afecta al cambio en las formas de enseñar y aprender en el contexto universitario.

Los profesores J. Salinas, de la Universidad de las Islas Baleares y la profesora V. I. Marín de la Universidad de Oldenburg en el artículo Las diferentes concepciones de la universidad digital en Iberoamérica nos presentan un estudio sobre el concepto 
de "universidad digital" desarrollado a través del análisis semántico de documentos publicados en diversos catálogos y bases de datos del ámbito Iberoamericano en el periodo 2007-2017.

Los profesores Cabero y Fernández, el primero de la Universidad de Sevilla y la segunda de la Universidad Isabel I, nos presentan en su artículo titulado Las tecnologías digitales emergentes entran en la Universidad: $R A$ y $R V$ un estudio basado en la revisión bibliográfica en torno a cómo se implementan y utilizan las tecnologías de la realidad aumentada y la realidad virtual en la enseñanza universitaria.

El artículo Uso de los registros de actividad para mejorar la colaboración en línea está firmado por los profesores de la Universidad de Barcelona, C. Coll y A. Engel, junto con Niño Carrasco de la Universidad de Baja California. En el mismo realizan una revisión teórica de publicaciones académicas sobre las herramientas y los procesos de utilización de los datos digitales registrados en entornos formativos en línea o de eLearning donde se entremezclan los conceptos de analíticas del aprendizaje, minería de datos y herramientas de análisis de interacciones.

Por su parte, las profesoras A. García-Valcárcel; González Rodero; Basilotta; y M. Martín de la Universidad de Salamanca presentan en su artículo REUNI+D: una red universitaria para la construcción colaborativa de conocimiento un estudio basado en encuesta sobre las visiones o representaciones de los investigadores responsables de grupos de investigación universitarios integrados en la Red Universitaria de Investigación e Innovación Educativa (REUNI+D) donde se explora los potenciales efectos de la misma para la construcción conjunta de conocimiento por parte de los miembros implicados.

Finaliza el monográfico con un artículo de Area, Sannicolás y Sanabria de la Universidad de La Laguna denominado Las aulas virtuales en la docencia de una universidad presencial: la visión del alumnado donde nos ofrecen un estudio empírico de aulas virtuales creadas como recursos de apoyo en la docencia presencial incorporando las perspectivas del alumnado sobre las mismas.

En definitiva, el lector encontrará en este número distintas perspectivas que abordan dónde nos encontramos en este proceso de tránsito desde un modelo decimonónico de docencia universitaria basada en la lección magistral y los apuntes, en una organización del tiempo y espacio académico regulada por horarios rígidos, y en la reproducción del conocimiento por los estudiantes, hacia una universidad integrada en el ecosistema digital que tiene la responsabilidad social y educativa de formar a la futura ciudadanía como sujetos profesionalmente competentes, cultos, emocionalmente equilibrados, con valores democráticos e integrados socialmente en el tiempo que les toca vivir. 


\section{NOTAS}

1. Precisamente en 2018 la Universidad de Salamanca celebra sus 800 años de su creación ocurrida en 1218.

2. https://www.4icu.org/about/index.htm

\section{REFERENCIAS BIBLIOGRÁFICAS}

Castell, M. (1999). La era de la información. Economía. Sociedad y Cultura, Vol 1. La sociedad red. Madrid: Alianza Editorial.
Postman, N. (1994). Tecnópolis. La rendición de la cultura a la tecnología. Barcelona: Galaxia Gutenberg/Círculo de Lectores.

\section{PERFIL ACADÉMICO Y PROFESIONAL DEL AUTOR}

Manuel Area Moreira. Doctor en Pedagogía y Catedrático de Didáctica y Organización Escolar de la Universidad de La Laguna. España.

E-mail: manarea@ull.edu.es

\section{Como citar este artículo:}

Area Moreira, M. (2018). Hacia la universidad digital: ¿dónde estamos y a dónde vamos? RIED. Revista Iberoamericana de Educación a Distancia, 21(2), pp. 25-30. doi: http://dx.doi.org/10.5944/ried.21.2.21801 